
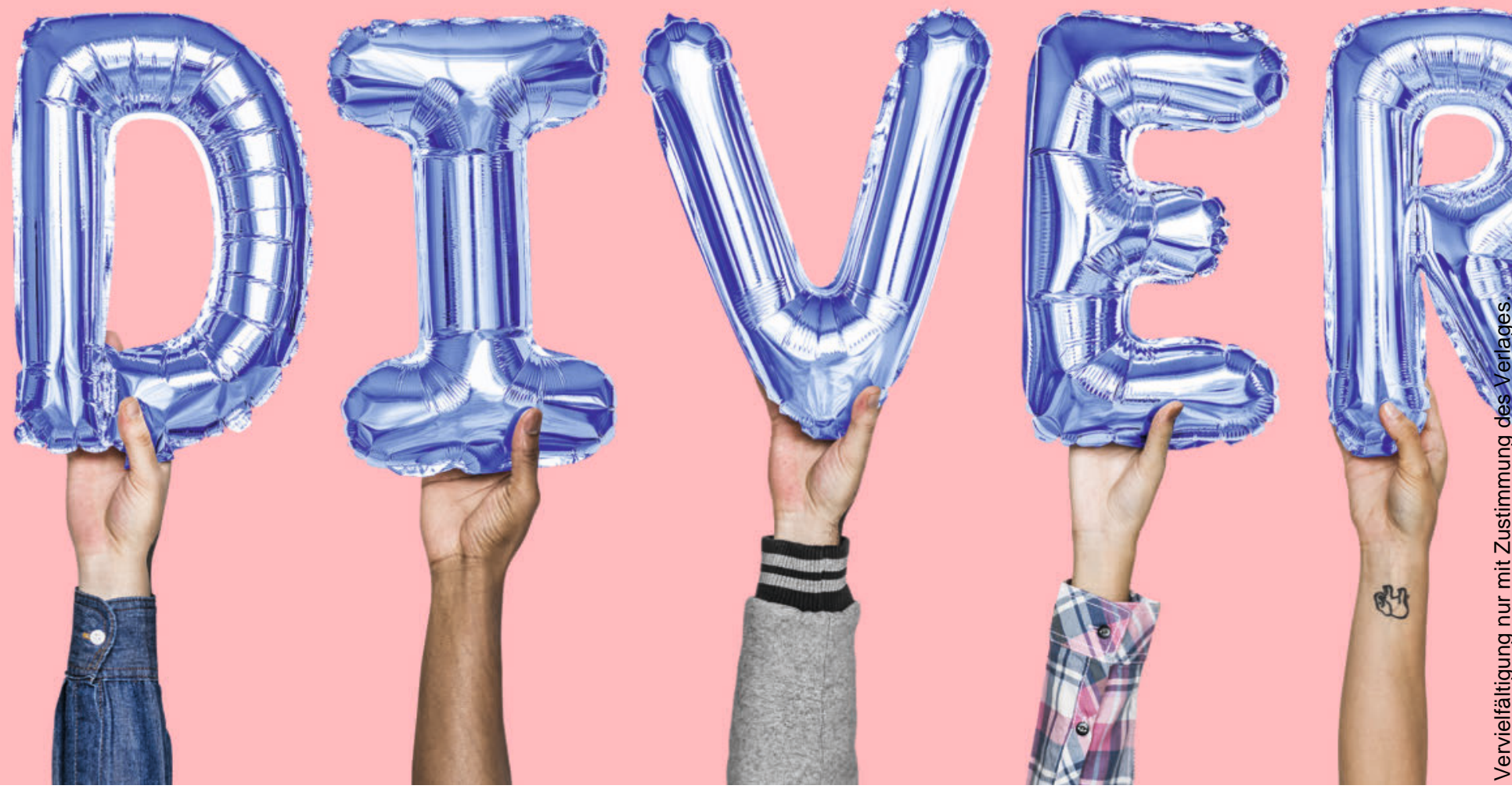

\title{
Werden wir mit unseren Modellen allen Klienten gerecht?
}

\begin{abstract}
Kulturelle Vielfalt Ergotherapeutische Modelle strukturieren unser Denken und unsere Arbeit. Sobald wir mit Klienten aus einem anderen Kulturraum arbeiten, stoßen wir jedoch an unsere Grenzen. Das ist wenig verwunderlich, da viele unserer Modelle, Theorien und Forschungsarbeiten in der westlichen Kultur entwickelt wurden - meist von gesunden weißen Frauen.
\end{abstract}

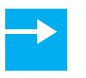

Unsere Gesellschaft wird immer multikultureller. Das liegt unter anderem an der großen Anzahl Geflüchteter und zunehmender Migration [1]. Schon seit längerer Zeit arbeiten Ergotherapeuten mit Klienten mit einem diversen Hintergrund und suchen fortlaufend nach Wegen, wie sie ihr ergotherapeutisches Angebot auf deren Bedarf abstimmen und optimal einsetzen können. Dabei stellt sich die Frage: Ist die Ergotherapie ausreichend darauf vorbereitet, die kulturelle Diversität in Ausbildung, Praxis und Forschung zu integrieren und sich an eine fortlaufend verändernde Gesellschaft anzupassen [2]?
Struktur für die Praxis $\rightarrow$ Um diese Frage beantworten zu können, werfen wir zunächst einen Blick auf die Modelle, die derzeit in der praktischen Arbeit zum Einsatz kommen. Dazu steht uns eine große Anzahl an sowohl ergotherapeutischen als auch nicht ergotherapeutischen Modellen zur Verfügung. Sie strukturieren unser Denken und Handeln und werden als Eckpfeiler der therapeutischen Praxis gesehen [3].

Diese konzeptionellen Modelle und Prozessmodelle [3]

$\rightarrow$ bieten unter anderem eine Struktur für den Therapieverlauf oder eine Brille, durch die man Klienten betrachten kann,

$\rightarrow$ unterstützen den Professional-ReasoningProzess,

$\rightarrow$ legen den Fokus auf die Interaktion von Klient (als Individuum, Gruppe, Organisation, Community, Population), Betätigung und Umwelt,

$\rightarrow$ gehen davon aus, dass sich bedeutungsvolle Betätigung auf Gesundheit und Wohlbefinden auswirkt und zu Partizipation führt,

$\rightarrow$ basieren neben weiteren Bezugswissenschaften unter anderem auf der Occupational Science (= Betätigungswissenschaft). 

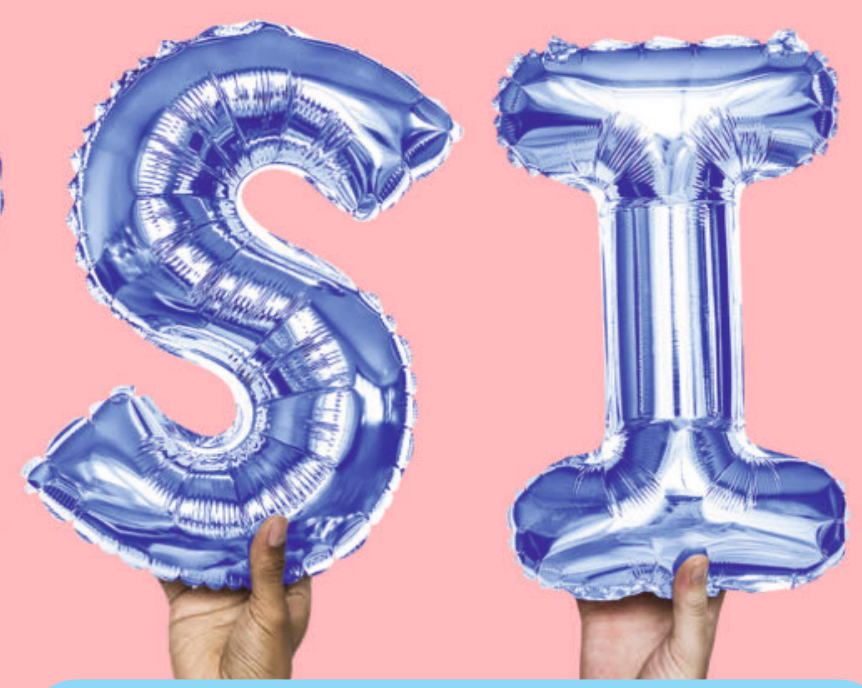

\section{Beispiel 1}

Eine junge türkische Frau mit Querschnittlähmung geht zur Behandlung in ein Rehazentrum. Die Ergotherapeutin bespricht mit ihr, was sie gerne möchte, und unterstützt sie dabei, den Alltag selbstständig zu meistern. Davon ist die Klientin angetan, und sie sieht die Möglichkeit, wieder ein Teil der Gesellschaft zu werden. Bis zu diesem Zeitpunkt wohnte sie mit ihren jüngeren Geschwistern bei den Eltern. Neben ihrem Sozialarbeitsstudium unterstütze sie ihre Mutter im Haushalt. Das interprofessionelle Team bereitet sie nun darauf vor, selbstständig in einem angepassten Appartment zu wohnen, ihr Studium wiederaufzunehmen und mit dem Auto bequem zur Uni zu fahren. Während ihrer Rehabilitation wohnte sie in einer angepassten Wohnung auf dem Gelände des Rehazentrums, da das Haus ihrer Eltern nicht rollstuhlgerecht war. Nach drei Monaten besucht sie ihre Eltern und erzählt voller Enthusiasmus von ihren Zukunftsplänen. Diese reagieren sehr negativ und wollen, dass sie sich entscheidet: zwischen einem selbstständigen Leben, das den Ausschluss aus der Familie bedeutet, oder der Rückkehr und von der Familie versorgt werden. Nach langem Zweifeln entscheidet sich die Klientin für ihre Familie. Das Therapieteam ist enttäuscht, weil alle Anstrengungen umsonst waren. Es hatte versäumt, die Klientin als Teil eines Systems zu sehen und ihre Kultur zu berücksichtigen.

\section{Beispiel 2}

Eine 35 Jahre alte Asylsuchende aus Syrien kommt über ein spezielles Programm in die Ergotherapie. Sie ist depressiv und wartet seit Jahren erfolglos auf eine Aufenthaltsgenehmigung. Die Ergotherapeutin plant für die erste Einheit ein Gespräch mittels OPHI, um sich ein Bild von ihr machen zu können. Die Klientin kommt nur schwer ins Gespräch, versteht vieles nicht und hat keine Ahnung, was all diese Fragen sollen. Als die Therapeutin spürt, dass sie so nicht weiterkommt, versucht sie herauszufinden, was die Klientin gerne in ihr Freizeit machen möchte, früher einmal gemacht hat, was sie gearbeitet hat etc. Auch dies alles ohne Erfolg. Daraufhin erzählt sie dem beratenden Coach des Asylzentrums enttäuscht von ihren Erfahrungen. Dieser kommt zusammen mit einem Dolmetscher in die nächste Therapieeinheit und fragt die Klientin, was sie in der Ergotherapie möchte. Dadurch wird klar, dass sie nur eines will: eine Aufenthaltserlaubnis. Ein anderes Ziel hat sie nicht.
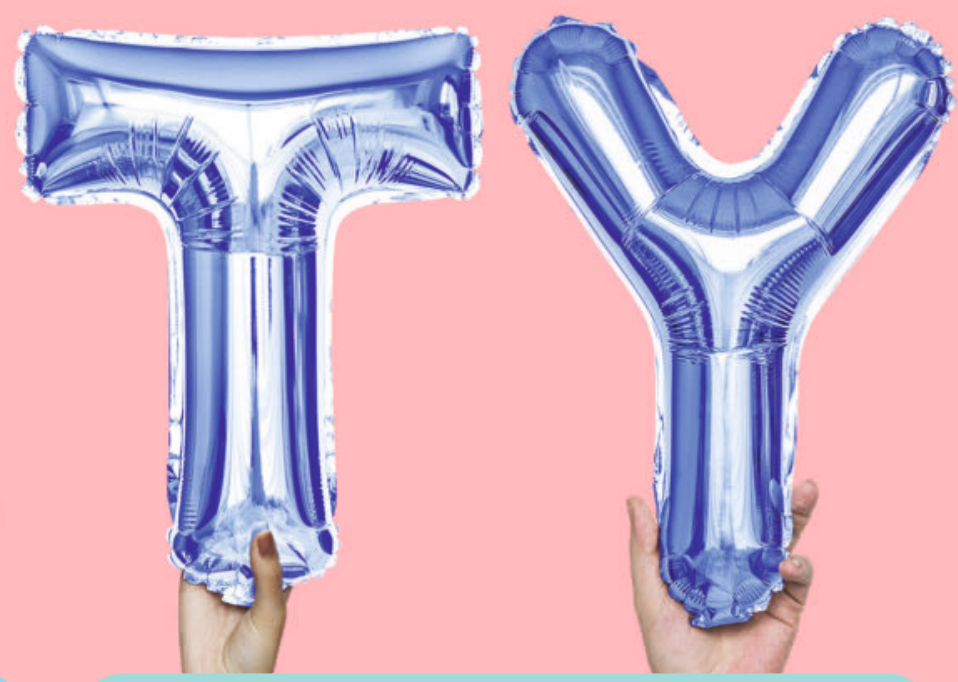

\section{Beispiel 3}

Ein 75-jähriger Herr indonesischer Herkunft mit einer Hemiplegie besucht die Ergotherapie. Die Therapeutin fragt ihn, ob er wieder selbstständig seine ADL durchführen möchte, und führt verschiedene Assessments mit ihm durch. Der Klient stimmt allem zu, und gemeinsam machen sie sich an die Arbeit. Nach einiger Zeit wird klar, dass er keinerlei Fortschritte macht. Während eines Hausbesuchs zur Wohnraumanpassung erzählt seine Frau, dass sie starke Rückenschmerzen hat und sehr darunter leidet. Auf Nachfrage der Ergotherapeutin berichtet sie, dass sie, wenn ihr Mann am Wochenende nach Hause kommt, alles für ihn macht. Sie trägt ihn vom Rollstuhl ins Bett, wäscht ihn, kleidet ihn an und füttert ihn. Im Gespräch mit dem Ehepaar wird deutlich, dass dies in der indonesischen Kultur gebräuchlich ist und der Mann das von seiner Frau erwartet. Aus Respekt gegenüber der Therapeutin hat er zu all ihren Vorschlägen „Ja“ gesagt, aber den Sinn der Therapie nicht verstanden.

\section{Beispiel 4}

Eine Bevölkerungsgruppe in den Niederlanden kommt ursprünglich aus Surinam, einem kleinen Staat an der Nordostküste Südamerikas. Erkranken Angehörige dieser Gruppe psychisch, werden sie - wie andere niederländische Bürger auch - an die Psychiatrie oder ein psychosoziales Institut verwiesen. Die Interventionen erzielen jedoch bei dieser Bevölkerungsgruppe kaum Erfolge, und den Klienten wird nicht geholfen. Auch Ergotherapeuten arbeiten mit Menschen aus Surinam, und zwar so, wie sie es auch bei ihren anderen Klienten gewohnt sind. Allerdings ebenfalls ohne Erfolg. Der Grund: Viele Surinamer leben den Winti-Glauben, eine Religion und Lebensart. Sie glauben unter anderem an übernatürliche Wesen. In ihrem Land würden Menschen mit einer psychiatrischen Erkrankung zu einem Bonoeman gehen, einem traditionellen Heiler, der gemäß dem Winti-Glauben mit Ritualen, Heilkräutern und Steinen arbeitet. Das heißt, in diesen Fällen helfen die Einteilung nach DSM-IV-Kriterien, ergotherapeutische Modellen und Assessments nicht weiter. Untersuchungen haben gezeigt, dass die Verbindung „regulärer“ Interventionen mit einem Bonoeman zu Zufriedenheit und Erfolg für die Klienten führt. Inzwischen gibt es in den Niederlanden ungefähr 150 anerkannte Bonoemänner, die bei der Behandlung einer psychiatrischen oder psychosozialen Problematik unterstützend hinzugezogen werden können. 
Bedarf an einem neuen Paradigma $\rightarrow$ Die ergotherapeutischen Modelle haben sich im Laufe der Zeit den Veränderungen der Gesellschaft, des Gesundheitswesens und des Berufes angepasst. In dieser Zeit hatte jedes Paradigma seine eigenen Theorien und Modelle - entsprechend dem jeweiligen Denken und der jeweiligen politischen Situation. In der ergotherapeutischen Literatur wird von drei Paradigmen gesprochen [4]:

\section{D.}

\section{Modelle und Theorien wurden aus westlicher Perspektive entwickelt.}

$\rightarrow$ das Paradigma der Betätigung (ca. 19201950)

$\rightarrow$ das mechanistische oder reduktionistische Paradigma (ca. 1950-1980)

$\rightarrow$ das heutige Paradigma (seit ca. 1980)

International wächst der Bedarf an einem neuen Paradigma. Es soll den Fokus verstärkt auf eine diversere Gesellschaft und auf interprofessionelle Zusammenarbeit mit nicht medizinischen Arbeitsfeldern legen. Zum Beispiel Architektur, Städteplanung, Politik, aber auch mit Gemeinden und der Technologie.

Von der westlichen Kultur geprägt $\rightarrow$ Derzeit kommen in der Regel ergotherapeutische Theorien und Modelle westlicher Herkunft zum Einsatz [2]. Sie wurden meist von erwachsenen weißen Frauen ohne Beeinträchtigung aus der Mittelklasse entwickelt, die im nordwestlichen Teil der Welt aufgewachsen sind, Englisch sprechen und in der Regel aus westlicher Perspektive denken und handeln. Auch die überwiegende Forschung findet durch Kollegen mit demselben Hintergrund im nordwestlichen Teil der Welt statt [2].

Wenn man bedenkt, dass ca. 80 Prozent der Menschen mit einer Beeinträchtigung nicht in diesem Teil der Welt leben, wird klar, dass ergotherapeutische Modelle und Assessments bei Klienten mit einem diversen kulturellen Hintergrund nicht immer passen können [5]. Dessen sind wir uns im Berufsalltag jedoch häufig nicht bewusst.

Tatsächlich können Menschen aus anderen Teilen der Welt einen ungewohnten Blick auf die Welt haben, anders über Gesundheit und Krankheit denken sowie unterschiedliche Normen und Werte vertreten. Sie sind möglicherweise von ihrer Religion beeinflusst, anders sozialisiert, an ein anderes Gesundheitssystem gewohnt, haben ungewohnte gesellschaftliche Gebräuche sowie einen für uns ungewöhnlichen sozialen, ökonomischen und politischen Status.

Kritisch hinterfragen $\rightarrow$ Wir sollten dafür sensibilisiert sein, dass die gängigen Theorien und Modelle von der westlichen Kultur geprägt sind, und sie daher kritisch hinterfragen.

Die anglokanadische Ergotherapeutin Karen Whalley Hammell hat innerhalb der Ergotherapie bahnbrechende Arbeit geleistet und mit ihrem kritischen Blick sehr zum Nachdenken angeregt. In vielen Publikationen und Vorträgen warf sie beispielsweise die Frage auf, warum Ergotherapie wichtig sein soll für unsere Gesellschaft. Ihr Ziel war es, eine wichtigere, relevantere und wertvollere Ergotherapie für die Gesellschaft zu gestalten.

Das „Social Model of Disability“ [6, 7] war für Hammell eine Offenbarung und unterstützte ihre Auffassung, dass die Probleme, mit denen Menschen mit einer Beeinträchtigung konfrontiert sind, mehr mit ihren ungleichen Möglichkeiten und Ressourcen als mit ihren körperlichen Bedingungen zu tun haben.

„Kritisches Denken und neue Perspektiven schaffen“ ist das übergeordnete Ziel ihrer Arbeit. Kritisches Denken ist wesentlich, um evidenzbasierte, sozial relevante und kulturell sichere Ergotherapiepraxis, -forschung, -theorien und -ausbildung zu entwickeln.

\section{Dy \\ 80\% der Menschen mit Beeinträchtigung leben nicht in der westlichen Welt.}

Philosophen behaupten, dass jeder Mensch ohne kritisches Denken unbewusst in ein bestehendes System integriert wird und sich den Ideen und Praktiken anpasst, die den Status quo aufrechterhalten [8]. Im Gegensatz dazu versuchen kritische Denker, den Status quo in Frage zu stellen. Sie möchten aufdecken, warum er entsteht und inwiefern er bestimmten sozialen Gruppen zugutekommt oder sie benachteiligt [9]. Daher möchte ich dafür plädieren, Philosophie als Bestandteil des ergotherapeutischen Unterrichts aufzunehmen. Studierende sollen lernen, kritisch und aus verschiedenen Perspektiven zu denken.

\section{5 \\ Ohne kritisches \\ Denken gibt es keine Weiterentwicklung.}

Kulturspezifische Annahmen $\rightarrow$ Fast alle ergotherapeutischen Modelle umfassen die drei Betätigungsbereiche Selbstversorgung, Produktivität und Freizeit. Das gab unserem Beruf lange Zeit eine sinnvolle inhaltliche Struktur. Im Laufe der Zeit begann Whalley Hammell jedoch, diese drei Bereiche in Frage zu stellen. Sie erkannte, dass sie nicht für alle Klienten sinnvoll waren. In den Bauerngemeinschaften, in denen sie arbeitete, spielte Freizeit beispielsweise keine Rolle. Damit möchte sie deutlich machen, dass es viele unangefochtene kulturspezifische Annahmen in unserem Beruf gibt, die exportiert werden, als würden sie „Wahrheiten“ darstellen [10].

\section{Verbesserung von Gesundheit erfordert} Verbesserung der Menschenrechte $\rightarrow$ Viele Ergotherapie-Theoretiker gehen davon aus, dass Menschen ihre täglichen Aktivitäten auswählen und gestalten. Aus einer westlichen Perspektive ist das sehr gut nachvollziehbar. Aber sind wir uns darüber bewusst, dass dies eindeutig ein Kennzeichen für lediglich einen Teil der Weltbevölkerung ist? Die Mehrheit der Menschen tut einfach, was zu tun ist, wozu sie gezwungen ist oder wozu sie die (eingeschränkte) Möglichkeit hat. Armut, Frauenfeindlichkeit, Patriarchate, Rassismus, Klassizismus und Altersdiskriminierung schränken die realen Möglichkeiten der Mehrheit der Weltbevölkerung ein, ihre Fähigkeiten einzusetzen [2].

Nicht nur Whalley Hammell, sondern auch andere Ergotherapeuten wie Townsend, Wilcock, Whiteford, Iwama, Kantartzis, Kronenberg, Ramugondo etc. sind davon überzeugt, dass die Verbesserungen von Gesundheit und Wohlbefinden nur mit Verbesserungen der Menschenrechte erreicht werden können. 

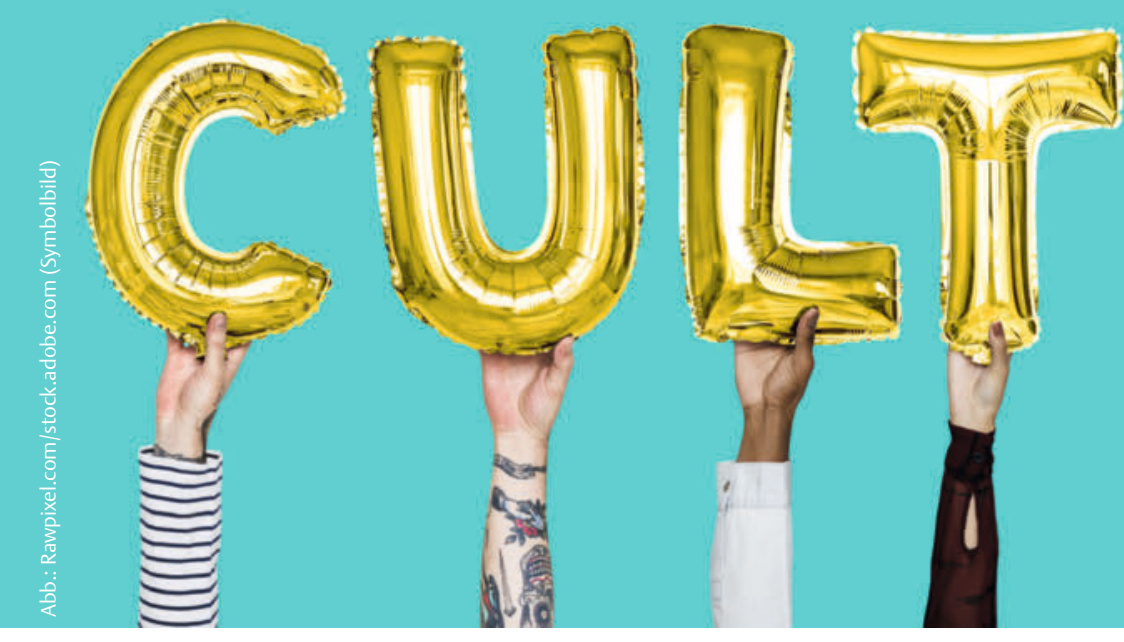
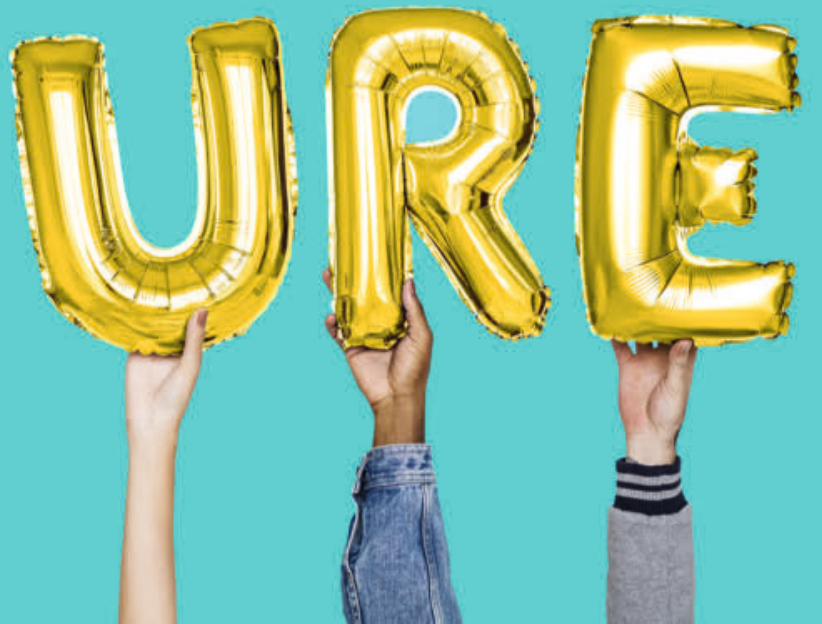

In den revidierten Mindeststandards für die Ausbildung von Ergotherapeuten legt der Weltverband WFOT im Jahr 2016 [11] bewusst den Fokus auf Menschenrechte. Die Frage ist, ob das bereits in den Curricula der Ergotherapieausbildungen angekommen ist.

\section{פ}

\section{Um einer diversen Gesellschaft gerecht $z u$ werden, müssen wir unseren Fokus ändern.}

Den Fokus ändern $\rightarrow$ Die griechisch-englische Ergotherapeutin Sarah Kantartzis [12] fragt sich, ob es ausreicht, was wir Ergotherapeuten aktuell unternehmen, um den Herausforderungen der Gesellschaft zu begegnen. Ihre Antwort: Nein. Wir sind auf dem Weg, aber noch lange nicht weit genug.

Die Gesellschaft hat unterschiedliche Erwartungen an uns und unsere Profession. Können wir sie erfüllen? Sarah Kantartzis meint, dass wir unseren Fokus ändern sollten: "Shifting our focus cause the lens we look through shapes the world we see“ [12]. Zum Beispiel indem wir neue Arbeitsplätze kreieren, wo Ergotherapeuten gefragt sind oder eine Rolle spielen können. Kantartzis stellt weitere Überlegungen an:

$\rightarrow$ Inwieweit sind Ergotherapeuten dazu imstande, sowohl die Systeme, in denen sie arbeiten, als auch ihre Rollen darin kritisch zu hinterfragen?

$\rightarrow$ Ist der aktuelle Arbeitsplatz, hauptsächlich innerhalb des Gesundheitssystems, der richtige Ort, um dem Bedarf einer diversen Gesellschaft und ihren zukünftigen Entwicklungen zu begegnen?

$\rightarrow$ Wir benötigen eine transdisziplinäre Herangehensweise mit verschiedenen Gruppen, Organisationen und Disziplinen.

$\rightarrow$ Wir benötigen andere Theorien und Modelle, um den Fragen einer diversen Gesellschaft begegnen zu können.

$\rightarrow$ Unser heutiger Wissensstand zu ergotherapeutischer Evidenz lässt sich nicht flächendeckend auf eine diverse Gesellschaft übertragen. Wir benötigen weitere Forschung.

$\rightarrow$ Sind unsere Vorgesetzten offen für Veränderungen? Es ist unsere Aufgabe, sie zu überzeugen, uns politisch zu engagieren, um als Berufsgruppe, Team oder als einzelne Therapeuten Einfluss auszuüben.

$\rightarrow$ Das bedeutet eine Veränderung für Ausbildung, Praxis und Forschung!

\section{Modelle aus Bezugswissenschaften nut-}

zen $\rightarrow$ Es gibt bereits Modelle und Theorien, die uns auf diesem Weg unterstützen könnten: $\rightarrow$ Social Model of Disability (z. B. Oliver, Barnes)

$\rightarrow$ Complex Systems Theories (z. B. Celliers, Morin, Woermann)

$\rightarrow$ Model of Occupational Empowerment for Marginalized Populations (z. B. Fisher, Hotchkiss)

$\rightarrow$ Participatory Occupational Justice Frame- work (z. B. Whiteford, Townsend)

$\rightarrow$ Recovery Model (z. B. Slade, Deegan)

$\rightarrow$ Ecological and Sustainable Models (z. B. Bronfenbrenner, Dunn)

$\rightarrow$ Health Promotion Models, Health Belief Models

$\rightarrow$ Change Models (z. B. Prochaska)

$\rightarrow$ Social/Economic Political Models (z. B. Dahlgren)

Jeder Anfang ist schwer und bringt Ungewissheit mit sich. Daher sind kritisches Denken und Handeln, Flexibilität und Offenheit für eine Vielzahl an Perspektiven gefragt!

Mieke le Granse

$\mathbf{a}$ Literaturverzeichnis

www.thieme-connect.de/products/ergopraxis $>$ „Ausgabe 2/20“

\section{(1) Autorin}

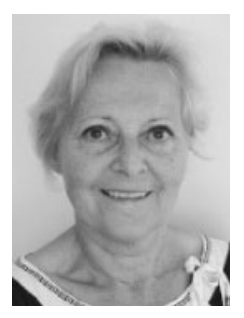

Mieke le Granse ist seit 1975 Ergotherapeutin. Die Niederländerin koordinierte bis 2017 den Bachelorstudiengang für deutsche Ergotherapeuten an der Zuyd Hogeschool in Heerlen. Sie ist Herausgeberin des Buches „Grundlagen der Ergotherapie“ - sowohl in der niederländischen als auch in der deutschen Version. 